\title{
A new proof of the uniqueness of the flow for ordinary differential equations with $B V$ vector fields
}

\author{
Maxime Hauray* and Claude Le Bris ${ }^{\dagger}$
}

March 2010

\begin{abstract}
We provide in this article a new proof of the uniqueness of the flow solution to ordinary differential equations with $B V$ vector-fields that have divergence in $L^{\infty}$ (or in $L^{1}$ ), when the flow is assumed nearly incompressible (see the text for the definition of this term). The novelty of the proof lies in the fact it does not use the associated transport equation.
\end{abstract}

Keywords :Ordinary differential equation, $B V$ vector-fields

AMS Classification : $34 \mathrm{~A} 12$

\section{Introduction and statement of our main result}

In 1989, P.-L. Lions and R. DiPerna showed in [10] the existence and the uniqueness of the almost everywhere defined flow solution to an ordinary differential equation of the type:

$$
\dot{y}(t)=b(t, y(t)),
$$

for $W^{1,1}$ vector fields $b$ with $L_{l o c}^{1}\left(\mathbb{R}_{t}, L_{y}^{\infty}\right)$ divergence (along with some technical assumptions). For such 'singular' vector fields, the only possibility is to solve the equation almost everywhere on the space $\Omega$ of initial conditions. In that case, one defines a flow $X(t, x): \mathbb{R} \times \Omega \rightarrow \Omega$ satisfying

$$
\left\{\begin{array}{l}
\dot{X}(t, x)=b(t, X(t, x)) \quad \text { for all } t \\
X(0, x)=x
\end{array} .\right.
$$

for almost all $x \in \Omega$. An initial time $s \neq 0$ may of course be chosen, and the flow then depends parametrically on this initial time $s$. With a view to simplifying the presentation, we will assume henceforth and throughout this article that the field $b$ is time-independent. Our arguments may be modified to cover the time-dependent case.

In the present article, we also adopt a notion of almost everywhere flow solution similar to that of DiPerna and Lions. We denote by $\left(X(t, \cdot)_{\#} \lambda\right)(E)=\lambda(X(-t, E))$ the pushforward of the Lebesgue measure $\lambda$. In the sequel, the vector-field $b$ will always be assumed at least $L_{l o c}^{1}$.

\footnotetext{
${ }^{*}$ Laboratoire d'Analyse, Topologie, Probabilités, CNRS et Université de Provence, Technopôle ChâteauGombert, 39, rue F. Joliot Curie, 13453 Marseille Cedex 13, France. hauray@cmi.univ-mrs.fr

${ }^{\dagger}$ CERMICS, École Nationale des Ponts et Chaussées, 6 \& 8, avenue Blaise Pascal, 77455 Marne-La-Vallée Cedex 2 and INRIA Rocquencourt, MICMAC project, Domaine de Voluceau, B.P. 105, 78153 Le Chesnay Cedex, France. lebris@cermics.enpc.fr
} 
Definition 1 (Almost everywhere, nearly incompressible flows). An almost everywhere flow solution to (2) is a measurable function $X(t, x): \mathbb{R} \times \Omega \rightarrow \Omega$ satisfying the following conditions:

(i) For almost all $x \in \Omega$, the map $t \mapsto X(t, x)$ is a continuous solution to $\dot{\gamma}=b(\gamma)$ satisfying $\gamma(0)=x$ and

$$
\text { for almost all } x \in \Omega, \quad \forall t \in \mathbb{R}, \quad X(t, x)=x+\int_{0}^{t} b(X(s, x)) d s
$$

(ii) For all $t$, the measure $X(t, \cdot)_{\#} \lambda$ is absolutely continuous with respect to $\lambda$, and, for all $T>0$, there exists a time-dependent function $C(T)>0$ such that the following condition of near-incompressibility holds

$$
\forall T \in \mathbb{R}_{+}, \forall|t| \leq T, \quad \frac{1}{C(T)} \lambda \leq X(t, \cdot)_{\#} \lambda \leq C(T) \lambda,
$$

(iii) $X$ is a one-parameter transformation group, i.e. satisfies

$$
X(t, X(s, x))=X(s+t, x), \quad \text { for almost all } x \in \Omega, \forall s, t
$$

Remark 1. Di Perna and Lions originally define in [10] a flow solution with condition $(i)$ replaced by $X \in \mathcal{C}\left(\mathbb{R}, L^{1}\right)$ satisfies the ordinary differential equation (2) in the sense of distribution. Their definition is equivalent to ours. It is indeed shown in [10] that the original definition implies $(i)$, and it can be shown that conditions (ii) and (iii) together imply that $X \in \mathcal{C}\left(\mathbb{R}, L^{1}\right)$.

Remark 2. Condition (ii) is in particular satisfied if $b$ is sufficiently smooth, and $\operatorname{div}(b) \in$ $L^{\infty}$. In that case $C(t)=e^{\|\operatorname{div}(b)\|_{\infty}|t|}$ is convenient. Besides this case, for a class of ordinary differential equations coming from some particular types of hyperbolic equations, such as the Keyfitz-Krantzer system, $\operatorname{div}(b)$ is only $L^{1}$, but an estimate of the form (ii) may be established using a maximum principle. See the work [6] by Bressan for more details on these systems and that by L. Ambrosio, F. Bouchut and C. De Lellis [4] for a discussion on the relevance of condition (ii).

We now give a brief state-of-the-art survey on the theory of ordinary differential equations with vector fields of low regularity. The seminal work 10 by DiPerna and Lions has been complemented and extended notably by L. Ambrosio in [2]. Several other authors have made important contributions. We would like to specifically cite the work 13 by N. Lerner which has inspired our own, present work. To date, the minimal conditions that are known to guarantee the existence and the uniqueness of the flow are the $B V$ regularity of the vector field, a $L^{1}$ bound on the divergence together with a near-incompressibility condition (or more classically a condition of bounded divergence) of the type (ii). The classical proofs of such results are based upon the consideration of the associated transport equation, written either in the conservative form

$$
\frac{\partial u}{\partial t}+\operatorname{div}(b(x) u)=0
$$

or in the non-conservative form

$$
\frac{\partial u}{\partial t}+b(x) \cdot \nabla_{x} u=0
$$


both with the initial condition $u(0, x)=u_{0}(x)$. Remark that, for divergence-free fields, the two equations coincide. When the existence and the uniqueness of the solution to the transport equation is established, for any given initial condition, one deduces the same result for the a.e. flow solution to the ordinary differential equation. The key ingredient for the resolution of the transport equation is a commutation lemma (first stated in [10]), which states that

$$
\rho_{\varepsilon} * \operatorname{div}(b u)-\operatorname{div}\left(\left(b * \rho_{\varepsilon}\right) u\right) \underset{\varepsilon \rightarrow 0}{\longrightarrow} 0 \text { in } L^{1} .
$$

C. De Lellis and G. Crippa have recently given in [9] a new proof of the existence and uniqueness of the flow solution of (2), not using the the associated transport equation. Their very interesting approach provides regularity estimates for $W^{1, p}$ vector-fields with $p>1$ but seemingly fails for $W^{1,1}$ vector-fields, unfortunately. Very recently, the approach has been succesfully improved by P.-E. Jabin. In [12, this author extends the direct method by C. De Lellis and G. Crippa to the case of bounded SBV vector fields (with locally finite jump set for the $d-1$ Hausdorff measure) in any dimension, and also to two-dimensional $B V$ vector-fields that satisfy a particular, local assumption in the direction of the flow. In addition, in [], the same author, in collaboration with $\mathrm{N}$. Champagnat, has proved that, in the particular case of an ODE corresponding to the Newton equation of motion (that is, $\dot{X}=V, \dot{V}=F(X)$ ), there exists a flow solution if $F \in H^{3 / 4} \cap L^{\infty}$, and this flow is unique among the class of flows obtained by regularization of the problem.

Before we get to the heart of the matter, some comments are in order regarding our assumptions, which are slightly different from those of [9]. The important assumption in [9] is the bound from above for $t>0$ in (ii), in order to prevent contraction of the measure. In addition, the group structure (our assumption (iii)) is not required in [9]. In the present state of our understanding, such differences seem to us related to the different techniques of proof used in 9 and here. The need for (iii) is consistent with our previous work [11] where, in order for the proof of uniqueness to be performed, the group structure is necessary in the Lagrangian viewpoint, but not in the Eulerian viewpoint. The assumption on the group structure is also present in [10. In the present contribution, we need this assumption because we transform integrals back and forth in time (see our argument in Section 2 below). To end this discussion, we also note that Assumption (ii) may be slightly weakened if we are only interested by the uniqueness of the forward-in-time flow. In that case, we may replace (ii) by

$$
\left(i i^{\prime}\right) \quad \frac{1}{C(T)} \lambda \leq X(t, \cdot)_{\#} \lambda<<\lambda, \quad \forall t \in[0, T] .
$$

This is in contrast to the assumptions of [9], which uses a bound from above: $X(t, \cdot)_{\#} \lambda \leq C(T) \lambda$. This owes to the very strategy of our proof, which mostly uses the backward-in-time flow. For instance, the most important step (7) is performed on the backward flow. In any event, owing to the group property, the upper and lower bounds are closely related to one another. The two uniform bounds in (ii) are indeed necessary to conclude when the issue considered is the uniqueness of both the backward-in-time and the forward-in-time flows. We refer the reader to Remark 5 at the end of the proof for more details.

\subsection{Main result}

The purpose of this article is to give a new and direct proof of the uniqueness of the a.e. flow solution to (2) for $B V$ vector fields, without arguing on the associated transport equation. We 
adopt the approach already used in 11 for $W^{1,1}$ vector fields. Basically, the commutation lemma instrumental in the proof contained in this prior publication is replaced by another strategy of proof, namely the introduction of a second variable. This is explained in details in the next paragraph.

Our result is the following:

Theorem 1. Let b be a $B V$ vector field on the $N$-dimensional torus $\mathbb{T}^{N}$. If $\operatorname{div}(b) \in L^{1}$, then there exists at most one a.e. flow solution to (2), in the sense of Definition 1 .

Remark 3. In [7], B. Perthame and I. Capuzzo Dolcetta remarked that the assumption " $b \in$ $W^{1,1}$ " of the original work by DiPerna and Lions could be replaced by the weaker assumption "the symmetric part of $\mathrm{Db}$ is a matrix-valued $L^{1}$ function". This observation seems to not be valid for the present strategy of proof, and more generally in the BV case. The reason is, their argument is based on the use of radially symmetric regularization kernels, while the regularization kernels we use here for the BV case are typically anisotropic.

Remark 4. Theorem 1 states uniqueness of the flow. We do not know which condition, besides the general assumptions of Theorem 1, makes possible the proof of existence of a flow in the sense of Definition 1, using the approach developed here for uniqueness.

\subsection{Main idea of the proof}

To start with, we outline here the proof performed in details in the next section. As already said, the proof uses a technique introduced in [11. In that work, a smooth convolution kernel $\rho$, with normalized integral, is considered. It is then proved that for any two a.e. flows $X$ and $Y$ solutions to the ordinary differential equation with $W^{1,1}$ coefficients,

$$
\lim _{\varepsilon \rightarrow 0} \frac{d}{d t}\left(\iint|X(t, x)-Y(t, y)| \frac{1}{\varepsilon^{N}} \rho\left(\frac{x-y}{\varepsilon}\right) d x d y\right)=0 .
$$

Now, the limit of the integral is

$$
\frac{d}{d t}\left(\int|X(t, x)-Y(t, x)| d x\right)=0 .
$$

This shows that, for all $t$,

$$
\int|X(t, x)-Y(t, x)| d x=0,
$$

since this quantity vanishes at initial time. The uniqueness of the solution follows. Remark that the introduction of the extra-variable $y$ allows to perform the calculation without using the transport equation.

Our aim is to now modify the above approach and treat BV vector fields. For this purpose, we use a convolution kernel well adapted to the geometry of the flow and the possible singularities of the BV vector field under consideration. In short, we consider the regularization kernel

$$
\frac{1}{\varepsilon^{N}} \rho\left(x, \frac{x-y}{\varepsilon}\right) \text { with } \rho(x, z)=F_{0}\left(|U(x) z|^{2}\right) \operatorname{det} U(x) \text {, and } U(x)=I d+\gamma \eta(x) \otimes \eta(x) .
$$


Here, $F_{0}$ is a smooth function, $\gamma$ is a constant that will be sent to infinity, and $\eta$ is an approximation of the direction normal to the jumps of the measure $D b$. The purpose of such a construction is to have a regularization that decreases faster in the direction normal to the jumps. The idea of a direction-dependent regularization was first introduced by P.L. Lions in [14. N. Lerner introduced the specific position-dependent regularization used here in [13] with a view to simplifying the proof of uniqueness originally given by L. Ambrosio for the $B V$ case. His argument, however, is still based upon the equivalence with the transport equation. In the present paper, we combine his argument with the approach consisting in introducing a second variable, already employed in [1] for $W^{1,1}$ vector fields.

\section{Proof of Theorem 1}

This section is devoted to the proof of Theorem 1. We denote by $\mu_{1}(t, \cdot)\left(\right.$ resp. $\left.\mu_{2}(t, \cdot)\right)$ the $L^{\infty}$ density of the measure $X(-t, \cdot)_{\#} \lambda$ (resp. $Y(-t, \cdot)_{\#} \lambda$ ) with respect to $\lambda$.

Consider now the kernel

$$
\frac{1}{\varepsilon^{N}} \rho\left(x, \frac{x-y}{\varepsilon}\right),
$$

where $\rho$ is a smooth, compactly supported, function, from $\mathbb{T}^{N} \times \mathbb{T}^{N}$ to $\mathbb{R}^{+}$which we will make precise below. Assume in addition $\rho$ satisfies $\int \rho(x, z) d z=1$ for all $x$. Our aim is to estimate

$$
I_{\varepsilon}(t)=\frac{d}{d t}\left(\iint|X(t, x)-Y(t, y)| \frac{1}{\varepsilon^{N}} \rho\left(x, \frac{x-y}{\varepsilon}\right) \mu_{1}(t, x) \mu_{2}(t, y) d x d y\right) .
$$

where $X$ and $Y$ are two flow solutions to (2). In the sense of distributions,

$$
\lim _{\varepsilon \rightarrow 0} I_{\varepsilon}(t)=\frac{d}{d t}\left(\int|X(t, x)-Y(t, x)| \mu_{1}(t, x) \mu_{2}(t, x) d x\right) .
$$

This is established using the Lebesgue continuity of the functions $Y$ and $\mu_{2}$ at almost every point, along with the $L^{\infty}$ bound on $\mu_{1}$. Remark that the Lebesgue continuity may be used if the support of $\rho(x, \cdot)$ is not exceedingly stretched in one direction (more specifically, we should have some constant $c>0$ such that $\forall x \in \mathbb{T}^{N}, B\left(0, c^{-1}\right) \subset \operatorname{Supp} \rho(x, \cdot) \subset B(0, c)$, See [15] for more details). The kernel we shall use satisfies such a condition for all $\varepsilon>0$, even though in the limit of a vanishing $\varepsilon$, it is infinitely stretched. Our purpose is to show that the limit (6) is

$$
\lim _{\varepsilon \rightarrow 0} I_{\varepsilon}(t)=-\int|X(t, x)-Y(t, x)| \operatorname{div}(b)(x) \mu_{1}(t, x) \mu_{2}(t, x) d x .
$$

This will eventually prove the uniqueness of the flow solution to (2) using the bounds from below on $\mu_{1}$ and $\mu_{2}$ inferred from (ii). To this end, we first perform the change of variable $(x, y) \rightarrow(X(t, x), Y(t, y))$ in $I_{\varepsilon}(t)$, and then differentiate under the integral

$$
I_{\varepsilon}(t)=\frac{d}{d t}\left(\iint|x-y| \frac{1}{\varepsilon^{N}} \rho\left(X(-t, x), \frac{X(-t, x)-Y(-t, y)}{\varepsilon}\right) d x d y\right),
$$

which we write $I_{\varepsilon}(t)=I_{\varepsilon}^{1}(t)+I_{\varepsilon}^{2}(t)$, with

$$
\begin{aligned}
& I_{\varepsilon}^{1}(t)=-\iint \frac{|x-y|}{\varepsilon^{N}} \partial_{1} \rho\left(X_{-t}(x), \frac{X_{-t}(x)-Y_{-t}(y)}{\varepsilon}\right) \cdot b\left(X_{-t}(x)\right) d x d y \\
& I_{\varepsilon}^{2}(t)=-\iint \frac{|x-y|}{\varepsilon^{N+1}} \partial_{2} \rho\left(X_{-t}(x), \frac{X_{-t}(x)-Y_{-t}(y)}{\varepsilon}\right) \cdot\left(b\left(X_{-t}(x)\right)-b\left(Y_{-t}(y)\right)\right) d x d y
\end{aligned}
$$


with the notations $X_{t}(x)=X(t, x)$ and $Y_{t}(y)=Y(t, y)$. Then, we return to the original variables $(x, y)$

$$
\begin{aligned}
& I_{\varepsilon}^{1}(t)=-\iint\left|X_{t}(x)-Y_{t}(y)\right| \frac{1}{\varepsilon^{N}} \partial_{1} \rho\left(x, \frac{x-y}{\varepsilon}\right) \cdot b(x) \mu_{1}(t, x) \mu_{2}(t, y) d x d y \\
& I_{\varepsilon}^{2}(t)=-\iint\left|X_{t}(x)-Y(t, y)\right| \frac{1}{\varepsilon^{N+1}} \partial_{2} \rho\left(x, \frac{x-y}{\varepsilon}\right) \cdot(b(x)-b(y)) \mu_{1}(t, x) \mu_{2}(t, y) d x d y,
\end{aligned}
$$

and next use the change of variable $z=(y-x) / \varepsilon$

$$
\begin{gathered}
I_{\varepsilon}^{1}(t)=-\iint\left|X_{t}(x)-Y_{t}(x+\varepsilon z)\right| \partial_{1} \rho(x, z) \cdot b(x) \mu_{1}(t, x) \mu_{2}(t, x+\varepsilon z) d x d z \\
I_{\varepsilon}^{2}(t)=-\iint\left|X_{t}(x)-Y_{t}(x+\varepsilon z)\right| \partial_{2} \rho(x, z) \cdot \frac{(b(x)-b(x+\varepsilon z))}{\varepsilon} \ldots \\
\ldots \mu_{1}(t, x) \mu_{2}(t, x+\varepsilon z) d x d z
\end{gathered}
$$

We now need to estimate these two terms when $\varepsilon$ goes to zero. We begin with the easiest of the two, namely $I_{\varepsilon}^{1}$.

\section{Step 1: Limit of $I_{\varepsilon}^{1}$}

Because $\rho$ is smooth, $b \in L^{1}$, and almost all points are Lesbesgue points for the two functions $Y$ and $\mu_{2}$, we can use the Lebesgue dominated convergence theorem and obtain

$$
\lim _{\varepsilon \rightarrow 0} I_{\varepsilon}^{1}(t)=-\int\left|X_{t}(x)-Y_{t}(x)\right|\left(\int \partial_{1} \rho(x, z) d z\right) \cdot b(x) \mu_{1}(t, x) \mu_{2}(t, x) d x .
$$

Now

$$
\int \partial_{1} \rho(x, z) d z=\frac{d}{d x}\left(\int \rho(x, z) d z\right)=0,
$$

since $\int \rho(x, z) d z=1$, for all $x$. Thus,

$$
\lim _{\varepsilon \rightarrow 0} I_{\varepsilon}^{1}(t)=0
$$

The treatment for $I_{\varepsilon}^{2}$ is more elaborate and will necessitate several steps.

\section{Step 2: Bound for $I_{\varepsilon}^{2}$}

We now wish to pass to the limit $\varepsilon \rightarrow 0$ in (8). If $b$ were $W^{1,1}$, the limit could easily be identified. It would suffice to replace $(b(x+\varepsilon z)-b(x)) / \varepsilon$ by $\int_{0}^{1} D b(x+\theta \varepsilon z) \cdot z d \theta$ in (8), and next use the Lebesgue dominated convergence theorem. All this does not require making specific the convolution kernel $\rho$ (See below and [11). Owing to the presence of the singular part of $D b$, we have to argue more carefully.

To proceed further, we recall the following result:

Proposition 1. [from [5, Theorem 1.28, Corollary 1.29]] Let b be a BV vector-field on $\mathbb{T}^{N}$.

(i) The Radon-Nikodym decomposition of its derivative $D b$ writes

$$
D b=D^{a} b+D^{s} b, \quad \text { with } \quad D^{a} b<<\mathcal{L}^{N}, D^{s} b \perp \mathcal{L}^{N},
$$


where the superscript a stand for "absolute continuous part", and s stand for "singular" respectively. As $D^{a} b$ is absolutely continuous with respect to the Lebesgue measure, we write it

$$
D^{a} b=\partial^{a} b d x
$$

where $\partial^{a} b$ is a $L^{1}$ matrix-valued fonction.

(ii) In addition, the polar decomposition of the singular part $D^{s} b$ of the measure $D b$ writes

$$
D^{s} b=M^{s}\left|D^{s} b\right|,
$$

where $\left|D^{s} b\right|$ is the total variation of the matrix-valued measure $D^{s} b$, and $M^{s}$ a matrix-valued fonction, such that $\left|M^{s}(x)\right|=1,\left|D^{s} b\right|$-a.e (the norm used for $M^{s}$ is the norm induced on matrices by the Euclidian norm of $\left.\mathbb{R}^{n}\right)$.

In view of the above decomposition, we now claim that

$$
\begin{aligned}
\limsup _{\varepsilon \longrightarrow 0} \iiint_{0}^{1}\left|X_{t}(x)-Y_{t}(x+\varepsilon z)\right| \ldots & \\
\ldots \mid & \partial_{2} \rho(x, z) \cdot\left(\frac{b(x+\varepsilon z)-b(x)}{\varepsilon}-\partial^{a} b(x+\varepsilon \theta z) \cdot z\right) \mid \mu_{1}(t, x) \mu_{2}(t, x+\varepsilon z) d \theta d x d z \\
& \leq 2 C(t)^{2} \iint\left|\partial_{2} \rho(x, z) \cdot M^{s}(x) \cdot z\right| d\left|D^{s} b\right|(x) d z .
\end{aligned}
$$

For convenience, we denote by

$$
I_{\varepsilon, a}^{2}=-\iiint_{0}^{1}\left|X_{t}(x)-Y_{t}(x+\varepsilon z)\right| \partial_{2} \rho(x, z) \cdot \partial^{a} b(x+\varepsilon \theta z) \cdot z d \theta d x d z,
$$

in the left-hand side, and

$$
\bar{I}_{s}^{2}(t)=\iint\left|\partial_{2} \rho(x, z) \cdot M^{s}(x) \cdot z\right| d\left|D^{s} b\right|(x) d z
$$

in the right-hand side.

To prove our claim, we regularize $X, Y, \mu_{1}$ and $\mu_{2}$, using some smooth $X^{\alpha}, Y^{\alpha}, \mu_{1}^{\alpha}$ and $\mu_{2}^{\alpha}$. Next, we replace $(b(x+\varepsilon z)-b(x)) / \varepsilon$ by $\int_{0}^{1} D b(x+\theta \varepsilon z) \cdot z d \theta$ (an equality true for almost all $(x, z)$ ) and perform the change of variable $x^{\prime}=x+\varepsilon \theta z$ (we use it even for the measure $D b$ because this is a linear change of variable). We obtain

$$
\begin{aligned}
I_{\varepsilon}^{2, \alpha}(t):= & -\iint\left|X_{t}^{\alpha}(x)-Y_{t}^{\alpha}(x+\varepsilon z)\right| \partial_{2} \rho(x, z) \cdot \frac{(b(x+\varepsilon z)-b(x))}{\varepsilon} \ldots \\
& \ldots \mu_{1}^{\alpha}(t, x) \mu_{2}^{\alpha}(t, x+\varepsilon z) d x d z \\
= & -\iiint_{0}^{1}\left|X_{t}^{\alpha}(x)-Y_{t}^{\alpha}(x+\varepsilon z)\right| \partial_{2} \rho(x, z) \cdot D b(x+\theta \varepsilon z) \cdot z \ldots \\
& \ldots \mu_{1}^{\alpha}(t, x) \mu_{2}^{\alpha}(t, x+\varepsilon z) d x d z d \theta \\
= & -\iiint_{0}^{1}\left|X_{t}^{\alpha}(x-\varepsilon \theta z)-Y_{t}^{\alpha}(x+\varepsilon(1-\theta) z)\right| \ldots \\
& \ldots \partial_{2} \rho(x-\varepsilon \theta z, z) \cdot D b(x) \cdot z \mu_{1}^{\alpha}(t, x-\varepsilon \theta z) \mu_{2}^{\alpha}(t, x+\varepsilon(1-\theta) z) d x d z d \theta .
\end{aligned}
$$


Let us decompose $I_{\varepsilon}^{2, \alpha}$ in two parts, according to the above Proposition 1 .

$$
I_{\varepsilon}^{2, \alpha}(t)=I_{\varepsilon, a}^{2, \alpha}(t)+I_{\varepsilon, s}^{2, \alpha}(t),
$$

where

$$
\begin{aligned}
I_{\varepsilon, a}^{2, \alpha}(t) & =-\iiint_{0}^{1}\left|X^{\alpha}(t, x-\varepsilon \theta z)-Y^{\alpha}(t, x+\varepsilon(1-\theta) z)\right| \ldots \\
& \ldots \partial_{2} \rho(x-\varepsilon \theta z, z) \cdot \partial^{a} b(x) \cdot z \mu_{1}^{\alpha}(t, x-\varepsilon \theta z) \mu_{2}^{\alpha}(t, x+\varepsilon(1-\theta) z) d x d z d \theta \\
\left|I_{\varepsilon, s}^{2, \alpha}(t)\right| & \leq 2 C(t)^{2} \iiint_{0}^{1} \partial_{2} \rho(x-\varepsilon \theta z, z) \cdot M^{s}(x) \cdot z\left|D^{s} b\right|(x) d z d \theta
\end{aligned}
$$

where we have used that $\left|X^{\alpha}-Y^{\alpha}\right| \leq 2$ (as we work on the torus). And letting $\varepsilon$ going to zero, we obtain (10) for $X^{\alpha}, Y^{\alpha}$ and the $\mu_{i}^{\alpha}$. Then (10) is obtained letting $X^{\alpha}, Y^{\alpha}, \mu_{1}^{\alpha}$ and $\mu_{2}^{\alpha}$ approximate $X, Y, \mu_{1}$ and $\mu_{2}$, respectively.

The majoration (10) being established, we proceed as follows. Arguing as above for $I_{\varepsilon}^{1}$, that is using the smoothness of $\rho$ and the fact that almost every point is a Lebesgue point for $Y, \mu_{1}$ and $\mu_{2}$, we obtain

$$
\begin{aligned}
\lim _{\varepsilon \rightarrow 0} I_{\varepsilon, a}^{2}(t)= & \lim _{\varepsilon \rightarrow 0}-\iiint_{0}^{1}\left|X_{t}(x-\varepsilon \theta z)-Y_{t}(x+\varepsilon(1-\theta) z)\right| \ldots \\
& \ldots \partial_{2} \rho(x-\varepsilon \theta z, z) \cdot \partial^{a} b(x) \cdot z \mu_{1}(t, x-\varepsilon \theta) \mu_{2}(t, x+\varepsilon(1-\theta) z) d \theta d x d z \\
= & -\iint\left|X_{t}(x)-Y_{t}(x)\right| \partial_{2} \rho(x, z) \cdot \partial^{a} b(x) \cdot z \mu_{1}(t, x) \mu_{2}(t, x) d x d z \\
= & -\int\left|X_{t}(x)-Y_{t}(x)\right| R_{a}(x) \mu_{1}(t, x) \mu_{2}(t, x) d x,
\end{aligned}
$$

with $R_{a}(x)=\int \partial_{2} \rho(x, z) \cdot \partial^{a} b(x) \cdot z d z$. To calculate this term, we integrate by parts and use the property $\forall x \in \mathbb{T}^{N}, \int \rho(x, z) d z=1$

$$
\begin{aligned}
R_{a}(x) & =\sum_{i, j} \int \frac{\partial \rho}{\partial z_{i}}(x, z) \frac{\partial^{a} b_{i}}{\partial x_{j}}(x) z_{j} d z=\sum_{i, j} \frac{\partial^{a} b_{i}}{\partial x_{i}}(x) \int-\rho(x, z) \frac{\partial z_{j}}{\partial z_{i}} d z \\
& =-\sum_{i} \frac{\partial^{a} b_{i}}{\partial z_{i}}(x) \int \rho(x, z) d z=-\operatorname{div}^{a} b .
\end{aligned}
$$

So we have obtained

$$
\lim _{\varepsilon \rightarrow 0} I_{\varepsilon, a}^{2}=\int\left|X_{t}(x)-Y_{t}(x)\right| \operatorname{div}^{a} b(x) \mu_{1}(t, x) \mu_{2}(t, x) d x
$$

The next step consists in proving that the right-hand side of (10) may be chosen arbitrarily small.

\section{Step 3: A bound on the singular part}

In order to estimate the right hand side of (10), we now use a geometric information, namely, the special form of $M^{s}(x)$, proved by G. Alberti [1]. 
Theorem 2. [Alberti's rank one Theorem, [5, Theorem 3.94]] Let $b$ be a BV vector-field defined on $\mathbb{T}^{N}$, and write $D b=D^{s} b+D^{a} b$ the Radon-Nikodym decomposition of its gradient. Consider $D^{s} b=M^{s}\left|D^{s} b\right|$ the polar decomposition of the singular part as in Proposition 1 . Then, $M^{s}$ is of rank one $\left|D^{s} b\right|$-almost everywhere, that is, there exists two vector-valued functions $\xi_{b}$ and $\eta_{b}$, both $\left|D^{s} b\right|$-measurables, such that $\xi_{b}$ and $\eta_{b}$ are unit vectors $\left|D^{s} b\right|$-a.e. and satisfy

$$
M^{s}(x)=\xi_{b}(x) \otimes \eta_{b}(x), \quad\left|D^{s} b\right|-\text { almost everywhere, }
$$

where $\xi_{b} \otimes \eta_{b}$ denotes the linear map $z \mapsto\left\langle\eta_{b}, z\right\rangle \xi_{b}$.

Corollary 1. As a consequence, the singular part of the divergence is

$$
\operatorname{div}^{s} b=\langle\xi, \eta\rangle\left|D^{s} b\right| .
$$

If we assume that the divergence of $b$ belongs to $L^{1}$, it follows that

$$
\langle\xi, \eta\rangle=0, \quad\left|D^{s} b\right|-\text { almost everywhere, }
$$

a property that will be crucial in the sequel.

Using the decomposition provided by Theorem 2, we rewrite our bound in (10), which we denote $\bar{I}_{s}^{2}(t)$ in the sequel

$$
\bar{I}_{s}^{2}(t) \leq 2 C(t)^{2} \iint\left|\left\langle\partial_{2} \rho(x, z), \xi_{b}(x)\right\rangle\right|\left|\left\langle\eta_{b}(x), z\right\rangle\right| d\left|D^{s} b\right|(x) d z .
$$

In order to render the right-hand side arbitrarily small, we now make specific our convolution kernel $\rho$. We choose

$$
\rho(x, z)=F_{0}\left(|U(x) z|^{2}\right) \operatorname{det}(U(x)),
$$

where $F_{0}$ is a smooth, compactly supported, non negative function such that $\int_{\mathbb{R}^{N}} F_{0}\left(|z|^{2}\right) d z=1$, and $U$ is a smooth, matrix-valued function, such that $U(x)$ is an orientation preserving matrix for all $x$. Note that owing to the presence of the determinant, the integral of $\rho(x, \cdot)$ remains equals to one independently of $x$. The dilation matrix $U(x)$ is set to $U(x)=$ $I d+\gamma \eta(x) \otimes \eta(x)$ (with the notation $a \otimes b$ for the endomorphism $x \rightarrow\langle b, x\rangle a$ ), where $\eta$ is a smooth vector-valued function. On the jump of the measure $D b, \eta$ will be chosen later as an approximation of the direction normal to the jump set. The factor $\gamma$ will be chosen as large as possible. It may possibly depend upon $x$ and be large only on a neighbourhood of the singular set of the measure $D b$, but we for simplicity of the calculation we will not use that not essential possibility here.

The partial derivative of $\rho$ writes

$$
\partial_{2} \rho(x, z)=2 F_{0}^{\prime}\left(|U(x) z|^{2}\right)\langle U(x) z, U(x) \cdot\rangle \operatorname{det}(U(x)) .
$$

We use this in the bound on $\bar{I}_{s}^{2}(t)$ to obtain

$$
\bar{I}_{s}^{2}(t) \leq C \iint\left|F_{0}^{\prime}\left(|U(x) z|^{2}\right)\right|\left|\left\langle U(x) z, U(x) \xi_{b}(x)\right\rangle\right|\left\langle\eta_{b}(x), z\right\rangle|\operatorname{det}(U(x)) d| D^{s} b \mid(x) d z,
$$

where here and below $C$ denotes various irrelevant constants. To simplify this term, we perform the change of variable $z \rightarrow U(x) z$, and obtain

$$
\bar{I}_{s}^{2}(t) \leq C \iint\left|F_{0}^{\prime}\left(|z|^{2}\right)\right|\left|\left\langle z, U(x) \xi_{b}(x)\right\rangle\right|\left|\left\langle\eta_{b}(x), U^{-1} z\right\rangle\right| d\left|D^{s} b\right|(x) d z .
$$


We next intend to use the special form $U(x)=I d+\gamma(x) \eta(x) \otimes \eta(x)$, to bound from above the two scalar products. Let us first formally illustrate our argument, performing our calculation with $\eta=\eta_{b}$, as if $\eta_{b}$ were smooth. In this case,

$$
\left|\left\langle z, U(x) \xi_{b}(x)\right\rangle\right|=\left|\left\langle z, \xi_{b}(x)\right\rangle\right| \leq|z|,
$$

because $\left\langle\eta_{b}, \xi_{b}\right\rangle=0$ and $\xi_{b}$ has unit norm. For the second scalar product,

$$
\left|\left\langle\eta_{b}(x), U^{-1}(x) z\right\rangle\right|=\frac{1}{1+\gamma}\left|\left\langle\eta_{b}(x), z\right\rangle\right| \leq \frac{1}{1+\gamma},
$$

because $U^{-1}=\mathrm{Id}-\frac{\gamma}{1+\gamma} \eta_{b} \otimes \eta_{b}$.

Inserting these bounds in (14), we obtain

$$
\bar{I}_{s}^{2}(t) \leq \frac{C}{1+\gamma} \iint\left|F_{0}^{\prime}\left(|z|^{2}\right)\right|\left|D^{s} b\right|(x) d z \leq \frac{C\left(F_{0}, b\right)}{1+\gamma},
$$

where the constant $C\left(F_{0}, b\right)$ depends only of $F_{0}$ and $b$. It remains then to let $\gamma$ to infinity to obtain $\bar{I}_{s}^{2}(t)=0$ and conclude our (formal) proof.

We now modify the above formal argument using an approximation $\eta$ of $\eta_{b}$, instead of $\eta_{b}$ itself. First, we remark

$$
\begin{aligned}
\left|\left\langle z, U(x) \xi_{b}\right\rangle\right| & =\left|\left\langle z, \xi_{b}+\gamma\left\langle\xi_{b}, \eta\right\rangle \eta\right\rangle\right| \\
& \leq\left(1+\gamma\left|\left\langle\xi_{b}, \eta\right\rangle\right|\right)|z| \\
& \leq\left(1+\gamma\left|\left\langle\xi_{b}, \eta-\eta_{b}\right\rangle\right|\right)|z| \\
& \leq\left(1+\gamma\left|\eta-\eta_{b}\right|\right)|z|,
\end{aligned}
$$

where we have used $\left\langle\xi_{b}, \eta_{b}\right\rangle=0,\left|D^{s} b\right|$-a.e (from Corollary 1), and that $\xi_{b}, \eta_{b}$ are unit vectors. To bound the scalar product $\left|\left\langle\eta_{b}(x), U^{-1} z\right\rangle\right|$, we decompose $z$ in $z=z_{\eta}+z_{\perp}$, where $z_{\eta}$ is the projection of $z$ on $\mathbb{R} \eta$

$$
\begin{aligned}
\left|\left\langle\eta_{b}, U^{-1} z\right\rangle\right| & =\left|\left\langle\eta_{b}, z-\frac{\gamma}{1+\gamma}\langle\eta, z\rangle \eta\right\rangle\right| \\
& =\left|\left\langle\eta_{b}, z_{\perp}+\frac{1}{1+\gamma} z_{\eta}\right\rangle\right| \\
& \leq\left|\left\langle\eta_{b}-\eta, z_{\perp}+\frac{1}{1+\gamma} z_{\eta}\right\rangle\right|+\left|\left\langle\eta, z_{\perp}+\frac{1}{1+\gamma} z_{\eta}\right\rangle\right| \\
& \leq\left(\left|\eta_{b}-\eta\right|+\frac{1}{1+\gamma}\right)|z| .
\end{aligned}
$$

From (15) and (16) we deduce

$$
\left|\left\langle z, U(x) \xi_{b}\right\rangle\right|\left|\left\langle\eta_{b}, U^{-1} z\right\rangle\right| \leq\left(2\left|\eta-\eta_{b}\right|+\frac{1}{1+\gamma}+\gamma\left|\eta-\eta_{b}\right|^{2}\right)|z|^{2} .
$$


We insert this bound in (14) and obtain

$$
\begin{aligned}
\bar{I}_{s}^{2}(t) & \leq C \int\left(\left|\eta-\eta_{b}\right|+\frac{1}{1+\gamma}+\gamma\left|\eta-\eta_{b}\right|^{2}\right)\left(\int F_{0}^{\prime}\left(|z|^{2}\right)|z|^{2} d z .\right)\left|D^{s} b\right|(x) \\
& \leq C\left(F_{0}\right)\left(\frac{1}{1+\gamma}+(1+2 \gamma) \int\left|\eta-\eta_{b}\right|\left|D^{s} b\right|(x)\right)
\end{aligned}
$$

because the integral $\int F_{0}^{\prime}\left(|z|^{2}\right)|z|^{2} d z$ is fixed, and both $\eta_{b}$ and $\eta$ are unit vectors.

We finally show that

$$
\inf _{\gamma>0, \eta \text { smooth }}\left(\frac{1}{1+\gamma}+(1+2 \gamma) \int\left|\eta-\eta_{b}\right|\left|D^{s} b\right|(x)\right)=0 .
$$

To this end, we first choose $\gamma$ such that $1 /(1+\gamma)$ is small, and then construct a smooth function $\eta$, sufficiently close to $\eta_{b}$ on the support of $D^{s} b$ so that $(1+2 \gamma) \int\left|\eta-\eta_{b}\right|\left|D^{s} b\right|(x)$ is also small (use for that classical approximation theorem with respect to the Radon measure $D^{s} b$ ). Note that $\eta$ can be arbitrarily extended to the whole torus as its value outside the support of $D^{s} b$ is irrelevant. This concludes the proof of the convergence of the right-hand side of (10) to zero.

Step 4: Conclusion Collecting all the previous results, we obtain

$$
\begin{aligned}
\frac{d}{d t} \int\left|X_{t}(x)-Y_{t}(x)\right| & \mu_{1}(t, x) \mu_{2}(t, x) d x= \\
& -\int\left|X_{t}(x)-Y_{t}(x)\right| \operatorname{div}(b)(x) \mu_{1}(t, x) \mu_{2}(t, x) d x .
\end{aligned}
$$

where we have replaced $\operatorname{div}^{a}(b)$ by $\operatorname{div}(b)$, since we are dealing with vector fields $b$ having at least, divergence in $L^{1}$. If $\operatorname{div}(b) \in L^{\infty}$, then

$$
\frac{d}{d t} \int\left|X_{t}(x)-Y_{t}(x)\right| \mu_{1}(t, x) \mu_{2}(t, x) d x \leq C \int\left|X_{t}(x)-Y_{t}(x)\right| \mu_{1}(t, x) \mu_{2}(t, x) d x .
$$

Since the integral in the right hand side vanishes initially, we conclude that

$$
\int\left|X_{t}(x)-Y_{t}(x)\right| \mu_{1}(t, x) \mu_{2}(t, x) d x=0
$$

and finally that $X(t, \cdot)=Y(t, \cdot)$ a.e. in $x$ since the $\mu_{i}$ are bounded away from 0 . Note that, as usual, if only the solution at positive times if of interest, an assumption on the negative part $\operatorname{div}(b)^{-}$of the divergence suffices to conclude.

When only the weaker hypothesis $\operatorname{div}(b) \in L^{1}$ holds, we have to slightly adapt the above argument. We choose a smooth compactly supported function $\phi(x)$, insert a factor $\phi(X(t, x))$ in the integral (5) defining $I_{\varepsilon}$. We now estimate

$$
I_{\varepsilon}^{\phi}(t)=\frac{d}{d t}\left(\iint \phi\left(X_{t}(x)\right)\left|X_{t}(x)-Y_{t}(y)\right| \frac{1}{\varepsilon^{N}} \rho\left(x, \frac{x-y}{\varepsilon}\right) \mu_{1}(t, x) \mu_{2}(t, y) d x d y\right) .
$$

The above argument carries over to the present case. An equality similar to (19) is obtained

$$
\begin{aligned}
\frac{d}{d t} \int & \phi\left(X_{t}(x)\right)\left|X_{t}(x)-Y_{t}(x)\right| \mu_{1}(t, x) \mu_{2}(t, x) d x \\
& =-\int \phi\left(X_{t}(x)\right)\left|X_{t}(x)-Y_{t}(x)\right| \operatorname{div}(b)(x) \mu_{1}(t, x) \mu_{2}(t, x) d x
\end{aligned}
$$


which can also be written (using the change of variable $x=X_{t}(x)$ )

$$
\begin{aligned}
& \frac{d}{d t} \int \phi(x)\left|x-Y_{t}\left(X_{-t}(x)\right)\right| \mu_{2}\left(t, X_{-t}(x)\right) d x \\
& \quad=-\int \phi(x)\left|x-Y_{t}\left(X_{-t}(x)\right)\right| \operatorname{div}(b)\left(X_{-t}(x)\right) \mu_{2}\left(t, X_{-t}(x)\right) d x .
\end{aligned}
$$

We next define $u(t, x)=\left|x-Y_{t}\left(X_{-t}(x)\right)\right| \mu_{2}\left(t, X_{-t}(x)\right)$. Equation (21) holding for all $\phi$, it follows that

$$
\frac{\partial u}{\partial t}+\operatorname{div}(b)\left(X_{-t}(x)\right) u=0
$$

in the distributional sense. There is no derivative of $u$ with respect to $x$ in the equation, so that the variable $x$ is only a parameter. Since $\operatorname{div}(b) \in L^{1}$ and condition (ii) holds, we have

$$
\int_{x} \int_{0}^{T}|\operatorname{div}(b)(X(-t, x))| d t d x<+\infty
$$

for all time $T$. So that, for almost all $x, \int_{0}^{T}|\operatorname{div}(b)(X(-t, x))| d t<+\infty$. Therefore equation (22) is well-posed for almost all $x$, and since by construction its solution $u$ vanishes at initial time, it vanishes for all time: $u(t, x)=0$ for all $t$, a.e. in $x$. This concludes the proof: $X \equiv Y$.

Remark 5. Given the above argument, the reader may now understand that, when the uniqueness of only the flow for positive times is under study, the upper bound $X(t, \cdot)_{\#} \lambda \leq C(T) \lambda$, for times $t>0$ in (ii) of Definition 11 may be somehow relaxed. In that case, and as briefly announced in the introduction, we may only assume that

$$
\left(i i^{\prime}\right) \quad \frac{1}{C(T)} \lambda \leq X(t, \cdot)_{\#} \lambda<<\lambda, \quad \forall t \in[0, T] .
$$

where the symbol $<<$ means here absolutely continuous with respect to. The group property allows to equivalently state (composing the previous inequality with $X(-t, \cdot)$ ) that

$$
\left(i i^{\prime}\right) \quad \lambda<<X(-t, \cdot)_{\#} \lambda \leq C(T) \lambda, \quad \forall t \in[0, T] .
$$

In that case, we have

$$
0<\mu_{2}(t, x) \leq C(T), \quad \text { a.e. in } \quad x,
$$

and also $0<\mu_{2}(t, X(-t, x))$ almost everywhere in $x$ since, considering

$$
\left(i i^{\prime}\right), \quad \lambda(X(t, A)) \leq C(T) \lambda(A)
$$

for all mesurable set $A$. This suffices to show that, for all $t>0$, and almost everywhere in $x$, $u(t, x)=0$ and thus conclude $X(t, x)=Y(t, x)$ in the above argument.

Remark 6. As pointed out by L. Ambrosio in [3], the use of the Alberti rank one theorem can be circumvented. In our proof, it is possible to use the following, much simpler ingredient. For any matrix $M$, we have

$$
\inf _{\rho} \int|\langle M z, \nabla \rho(z)\rangle| d z=|\operatorname{Tr}(M)|,
$$


where the infimum is taken over all smooth kernels with total mass one. Essentially applying this result to the matrices $D^{s} b(x)$ we manipulate in the proof, and using $\operatorname{Tr}\left(D^{s} b(x)\right)=\operatorname{div}\left(b^{s}\right)(x)=0$, we may obtain an estimate analogous to (17), thus (18). We then conclude our argument similarly. We however believe that considering the Alberti rank one Theorem helps to better understand the geometry of the problem.

\section{Acknowledgements.}

This work was mostly completed while the second author was visiting the Institute for Mathematics and its Applications and the Department of Mathematics of the University of Minnesota. The hospitality of these institutions is gratefully acknowledged. Both authors would also like to thank Pierre-Emmanuel Jabin for helpful discussions.

\section{References}

[1] Giovanni Alberti. Rank one property for derivatives of functions with bounded variation. Proc. Roy. Soc. Edinburgh Sect. A, 123(2):239-274, 1993.

[2] Luigi Ambrosio. Transport equation and Cauchy problem for $B V$ vector fields. Invent. Math., 158(2):227-260, 2004.

[3] Luigi Ambrosio. Transport equation and Cauchy problem for non-smooth vector fields. Lecture Notes of the CIME Summer school in Cetrary, June 27-July 2, 2005, available at http://cvgmt.sns.it/papers/amb05/Cetrarotext.pdf, 2005.

[4] Luigi Ambrosio, François Bouchut, and Camillo De Lellis. Well-posedness for a class of hyperbolic systems of conservation laws in several space dimensions. Comm. Partial Differential Equations, 29(9-10):1635-1651, 2004.

[5] Luigi Ambrosio, Nicola Fusco, and Diego Pallara. Functions of bounded variation and free discontinuity problems. Oxford Mathematical Monographs. The Clarendon Press Oxford University Press, New York, 2000.

[6] Alberto Bressan. An ill posed Cauchy problem for a hyperbolic system in two space dimensions. Rend. Sem. Mat. Univ. Padova, 110:103-117, 2003.

[7] Italo Capuzzo Dolcetta and Benoit Perthame. On some analogy between different approaches to first order PDE's with nonsmooth coefficients. Adv. Math. Sci. Appl., 6(2):689703, 1996.

[8] Nicolas Champagnat and Pierre-Emmanuel Jabin. Well-posedness in any dimension for hamiltonian flows with non BV force terms. Accepted for publication in Comm. P.D.E., available at http://arxiv.org/abs/0904.1119, 2009.

[9] Gianluca Crippa and Camillo De Lellis. Estimates and regularity results for the DiPernaLions flow. J. Reine Angew. Math., 616:15-46, 2008.

[10] Ronald J. DiPerna and Pierre-Louis Lions. Ordinary differential equations, transport theory and Sobolev spaces. Invent. Math., 98(3):511-547, 1989. 
[11] Maxime Hauray, Claude Le Bris, and Pierre-Louis Lions. Deux remarques sur les flots généralisés d'équations différentielles ordinaires. C. R. Acad. Sci. Paris, 344:759-764, 2007.

[12] Pierre-Emmanuel Jabin. Differential equations with singular fields. Available at http://math1.unice.fr/ jabin/odefinal.pdf, 2009.

[13] Nicolas Lerner. Transport equations with partially $B V$ velocities. Ann. Sc. Norm. Super. Pisa Cl. Sci. (5), 3(4):681-703, 2004.

[14] Pierre-Louis Lions. Sur les équations différentielles ordinaires et les équations de transport. C. R. Acad. Sci. Paris Sér. I Math., 326(7):833-838, 1998.

[15] Elias M. Stein. Singular integrals and differentiability properties of functions. Princeton Mathematical Series, No. 30. Princeton University Press, Princeton, N.J., 1970. 